\title{
First report on trichomonads from true bugs
}

\author{
Pavla Smejkalová ${ }^{1,2}$, Jan Votýpka ${ }^{2,3}$, Julius Lukeš $\breve{3}^{3,4}$ and Ivan Čepička ${ }^{1}$ \\ ${ }^{1}$ Department of Zoology, Faculty of Science, Charles University in Prague, Prague, Czech Republic; \\ ${ }^{2}$ Department of Parasitology, Faculty of Science, Charles University in Prague, Prague, Czech Republic; \\ ${ }^{3}$ Biology Centre, Institute of Parasitology, Czech Academy of Sciences, České Budějovice, Czech Republic; \\ ${ }^{4}$ Faculty of Science, University of South Bohemia, České Budějovice, Czech Republic
}

\begin{abstract}
Although the hindgut of some insects represents a rich source of intestinal trichomonads, their diversity is only poorly understood. The aim of the present study was to investigate the presence and abundance of intestinal trichomonads in true bugs (Heteroptera). We microscopically examined intestinal contents of more than 780 specimens belonging to 28 families of true bugs from localities in China, Ghana and Papua New Guinea for the presence of intestinal endosymbionts. More than 120 samples were examined also by means of PCR using trichomonad-specific primers. We determined sequences of SSU rDNA and ITS region of two isolates of the genus Simplicimonas Cepicka, Hampl et Kulda, 2010 and one isolate of Monocercomonas colubrorum (Hammerschmidt, 1844). Although our results showed that trichomonads are very rare inhabitants of the intestine of true bugs, two of three isolated flagellates belong to species specific for reptiles. The possibility of transmission of trichomonads between reptiles and true bugs is discussed.
\end{abstract}

Keywords: Parabasalia, intestinal endosymbionts, Simplicimonas, Monocercomonas, phylogeny, SSU rDNA, Heteroptera, Insecta, host-parasite association

Trichomonads (Parabasalia) comprise flagellated protists belonging to the eukaryotic supergroup Excavata and the group Metamonada (see Cavalier-Smith 2002, 2003). Trichomonads are characterised by the presence of hydrogenosomes, which are highly modified mitochondria, parabasal apparatus composed of a Golgi complex attached to striated fibres, and closed pleuromitosis with an extranuclear spindle (Cepicka et al. 2010). The main life stage of trichomonads is a flagellated trophozoite and some species are known to produce pseudocysts with internalized flagella (Pereira-Neves et al. 2003), whereas true cysts have been reported only for a few species (Brugerolle 1973, Farmer 1993, Dolan et al. 2004, Hampl et al. 2007). Trichomonads are highly diversified and include approximately 450 species (Adl et al. 2007). With the exception of just a few described free-living species (Yubuki et al. 2010), most trichomonads are cooperative endosymbionts, commensals or parasites of various vertebrates or invertebrates.

Besides medically and veterinary important parasites, such as Trichomonas vaginalis Donné, 1836, Trichomonas gallinae (Rivolta, 1878), Tritrichomonas foetus (Riedmüller, 1928), Histomonas meleagridis (Smith, 1895) and ecologically important endosymbionts of lower termites and wood roaches, the diversity and phylogeny of other intestinal trichomonads remain considerably understudied. This is particularly true for trichomonads from non-termite insects. Several studies from the first half of the 20th century showed by means of light microscopy that larvae of beetles, caddisflies and dipterans harbour a rich diversity of trichomonads in their hindguts (Mackinnon 1910, 1912, Travis 1932, Kowalczyk 1938, Ludwig 1946). In contrast, only two molecular-phylogenetic studies concerning trichomonads of insects other than termites and cockroaches have been published so far (Zhang et al. 2003, McElroy et al. 2005)

The aim of the present study was to investigate the presence and abundance of intestinal trichomonads in true bugs (Heteroptera). Although our results show that trichomonads are very rare inhabitants of the intestine of true bugs, we identified trichomonad species identical with reptilian endosymbionts in two cases.

\section{MATERIALS AND METHODS}

\section{Sampling and culture conditions}

Numerous localities in China, Ghana and Papua New Guinea were sampled for true bugs in frame of a project studying the diversity of their trypanosomatids (Votýpka et al. 2010, 2012). True bugs were dissected in the field within 24 hours after the capture and their intestine was examined under the light microscope. When flagellates were detected, parts of the infected gut were transferred to preservation solution ( $1 \mathrm{ml}$ of $2 \% \mathrm{SDS}$, $100 \mathrm{mM}$ EDTA solution) and at the same time to cultivation medium (SNB-9 agar mixed with rabbit blood overlaid with SNB-9 
medium containing $10 \%$ fetal calf serum (Sigma-Aldrich, St. Louis, USA), $100 \mathrm{mg}$ gentamicin, $1000 \mathrm{U}$ penicillin and $1.5 \mathrm{mg}$ fluorocytosine). Two cultures containing trichomonads, $\mathrm{CH} 394$ and PNG73, were established. After transportation to the laboratory, axenic primary culture of the strain $\mathrm{CH} 394$ was transferred into Dobell and Laidlaw's biphasic medium (Dobell and Laidlaw 1926) with the addition of unidentified bacteria as well as into TYM medium (Diamond 1957). Cultures were maintained at room temperature and were subcultured once a week. Isolate PNG73 was introduced into Dobell and Laidlaw's biphasic medium with the addition of unidentified bacteria, but failed to grow after the third passage.

In addition, fixed intestinal contents of true bugs from Ghana and China were analysed by means of PCR using trichomonad-specific primers (see below). A total 124 samples of fixed intestinal contents of several species of true bugs were examined: 67 samples (G1-G38 and G40-G68) from Ghana (for information on samples see Votýpka et al. 2012) and 57 samples (CH 39, CH48-51, CH53, CH76-CH89, CH111, CH112, CH148, CH152, CH230-CH232, CH246, CH249, CH278CH282, CH300, CH304, CH306, CH322, CH332, CH334, CH338-342, CH380, CH387, CH390-CH392, CH394, CH395, CH402, CH404-CH406, CH412) from China (for information on samples see Votýpka et al. 2010).

\section{Light microscopy}

Light microscopical observations were performed using a BX51 microscope equipped with a DP71 camera (Olympus, Tokyo, Japan). Morphology of the strain CH394 in both culture media was examined in protargol-stained preparations. Moist films spread on cover slips were prepared from pelleted cultures obtained by centrifugation at $500 \mathrm{~g}$ for $8 \mathrm{~min}$. The films were fixed in Bouin-Hollande's fluid for $4 \mathrm{~h}$, washed with $70 \%$ ethanol and stained with $1 \%$ protargol (Bayer, Leverkusen, Germany) following the Nie's (1950) protocol.

\section{DNA isolation, amplification and sequencing}

The lysate of intestinal tissue and content in the preservation solution was used to isolate total DNA using High Pure PCR Template Preparation Kit (Roche, Basel, Switzerland). Genomic DNA from cultured flagellates was isolated using the DNeasy Blood \& Tissue Kit (Qiagen, Hilden, Germany) according to manufacturer's instructions. Primers 16Sl (TACTTGGTTGATCCTGCC; Tachezy et al. 2002) and 16SRR (TCACCTACCGTTACCTTG; Cepicka et al. 2005) were used to amplify the SSU rRNA gene. Primers ITSF (TTCAGTTCAGCGGGTCTTCC) and ITSR (GTAGGTGAACCTGCCGTTGG) (Cepicka et al. 2005) were used to amplify the ITS region.

Most amplicons were purified using the QIAquick PCR Purification Kit (Qiagen) and directly sequenced on the ABI Prism 3100-Avant Genetic Analyzer (Applied Biosystems, Foster City, USA). SSU rRNA gene of the isolate PNG73 was cloned into the pGEM ${ }^{\circledR}-\mathrm{T}$ EASY vector (Promega, Fitchburg, USA). Sequences of six clones of the SSU rRNA gene were determined and two most divergent ones, differing in 6 nucleotides from each other, were selected for the analysis. Sequence data reported in this paper are available in GenBank under accession numbers KJ101558-KJ101564.

\section{Phylogenetic analyses}

Dataset containing SSU rDNA sequences of Tritrichomonadida with the exclusion of long branch-forming Dientamoebidae was constructed. Sequences were aligned using the MAFFT method (Katoh et al. 2002) with the help of the MAFFT 6 server (http://mafft.cbrc.jp/alignment/server/index.html) using the G-INS-i algorithm at default settings. The resulting alignment was manually edited in BioEdit 7.0.9.0 (Hall 1999). The length of the final alignment was 1491 characters and it is available upon request. Phylogenetic trees were constructed by maximum likelihood and Bayesian methods. Maximum likelihood trees were constructed in RAxML 7.0.3 (Stamatakis 2006) under the GTRGAMMAI model. Bootstrap support values were generated using 1000 pseudoreplicates.

\section{RESULTS}

Microscopical examination of intestinal contents of more than 780 specimens of true bugs belonging to 28 families collected in China, Ghana and Papua New Guinea revealed that between 15 and 25\% samples (depending on the country and location) were microscopically positive for kinetoplastids; trichomonads were observed in only two true bugs. Inoculation of these two samples into the media resulted in the establishment of two strains, subsequently determined as members of the genus Simplicimonas Cepicka, Hampl et Kulda, 2010: strain CH394 isolated from Melamphaus faber (Fabricius) (Pyrrhocoridae) from Jinhong - Xishuangbanna, China $\left(22^{\circ} 04^{\prime} \mathrm{N}\right.$; $102^{\circ} 42^{\prime} \mathrm{E}$ ), and strain PNG73 obtained from Paraectatops costalis costalis (Walker) (Pyrrhocoridae) from Madang, Papua New Guinea (5ㅇ'23"S; $145^{\circ} 47^{\prime} 41$ "E). Culture of the strain $\mathrm{CH} 394$ was stable for more than 50 passages in Dobell and Laidlaw's biphasic medium and was successfully cryopreserved in liquid nitrogen in the presence of $5 \%$ dimethyl sulfoxide. Culture of the strain PNG73 failed to grow after the third passage. Sequences of the SSU rDNA gene and ITS region were determined for both strains.

To address the possibility of weak infection having been overlooked during microscopic examinations in the field, we additionally analysed 124 fixed samples of intestinal contents of true bugs collected in Ghana and China by means of PCR using trichomonad-specific primers. Only two samples were PCR-positive. We determined SSU rDNA sequence of Monocercomonas colubrorum (Hammerschmidt, 1844) from the sample G37 isolated from Rhynocoris bicolor (Fabricius) (Reduviidae) from Abrafo, Ghana $\left(5^{\circ} 20^{\prime} 29^{\prime \prime} \mathrm{N} ; 1^{\circ} 22^{\prime} 58^{\prime \prime} \mathrm{W}\right)$, and an SSU rDNA sequence falling into the genus Simplicimonas from the sample CH394 isolated from Melamphaus faber (Pyrrhocoridae) from Jinhong - Xishuangbanna, China $\left(22^{\circ} 04^{\prime} \mathrm{N} ; 102^{\circ} 42^{\prime} \mathrm{E}\right)$. The latter sequence was identical with that obtained from cultured strain $\mathrm{CH} 394$.

Phylogenetic tree of the SSU rDNA of Tritrichomonadida as defined by Cepicka et al. (2010), excluding the long branch-forming Dientamoebidae, is shown in Fig. 1. Strains CH394 and PNG73 were robustly placed within the genus Simplicimonas. Although the internal relationships within Simplicimonas remain unsupported, PNG73 formed its basal branch and CH394 appeared closely re- 


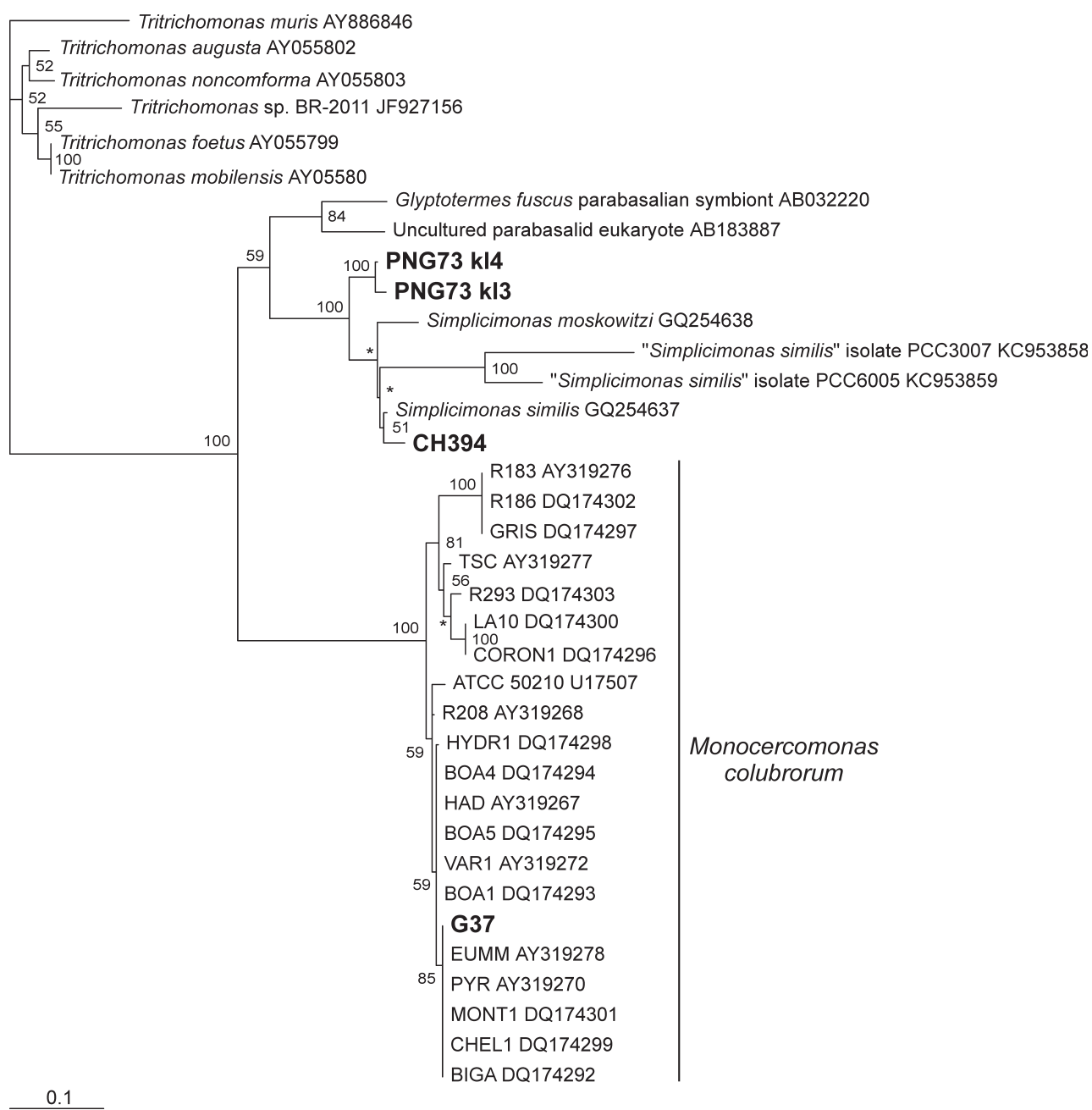

Fig. 1. Phylogenetic tree of Tritrichomonadida based on SSU rDNA. The tree was constructed by the maximum likelihood method in RAxML under the GTRGAMMAI model; the values at the nodes represent statistical support in bootstrap values (RAxML). Support values below $50 \%$ are represented by an asterisk $\left({ }^{*}\right)$. The newly obtained sequences are in bold.

Table 1. Uncorrected genetic distances (\%) between strains of Simplicimonas based on sequences of the ITS region.

\begin{tabular}{|c|c|c|c|c|}
\hline & $\mathrm{CH} 394$ & Simplicimonas similis $\mathrm{ULI}^{1}$ & Simplicimonas moskowitzi CRIST $1^{1}$ & PNG73 \\
\hline Simplicimonas similis $\mathrm{ULI}^{1}$ & 0.006 & & & \\
\hline Simplicimonas moskowitzi* ${ }^{*}$ CRIST $1^{1}$ & 0.015 & 0.021 & & \\
\hline PNG73 & 0.061 & 0.058 & 0.076 & \\
\hline Simplicimonas sp. GABC $1^{2}$ & 0.079 & 0.082 & 0.094 & 0.085 \\
\hline
\end{tabular}

${ }^{1}$ isolated from a lizard by Cepicka et al. (2010); ${ }^{2}$ isolated from a bird by Lollis et al. (2011); * Simplicimonas moskowitzi (Honigberg, 1963) isolated from a lizard by Cepicka et al. (2010)

lated to S. similis Cepicka, Hampl et Kulda, 2010. The genetic distance (uncorrected p-distance) between $\mathrm{CH} 394$ and $S$. similis was $0.6 \%$. Uncultured isolate G37 was placed into Monocercomonas colubrorum, specifically to its clade 3 as defined by Hampl et al. (2007), having identical SSU rDNA sequence with the strains MONT1, CHEL1, BIGA, PYR, and EUMM.

In addition, the sequence of the ITS region was determined for both Simplicimonas strains, even though phy- logenetic analysis failed to determine relationships within the genus Simplicimonas (data not shown). Uncorrected genetic distances between individual Simplicimonas strains are shown in Table 1.

Cells of the strain CH394 were stained by protargol and analysed by light microscopy. Morphology was examined in cells growing in two different culture media: polyxenic agnothobiotic culture in the Dobell and Laidlaw's biphasic medium, and axenic culture in TYM me- 


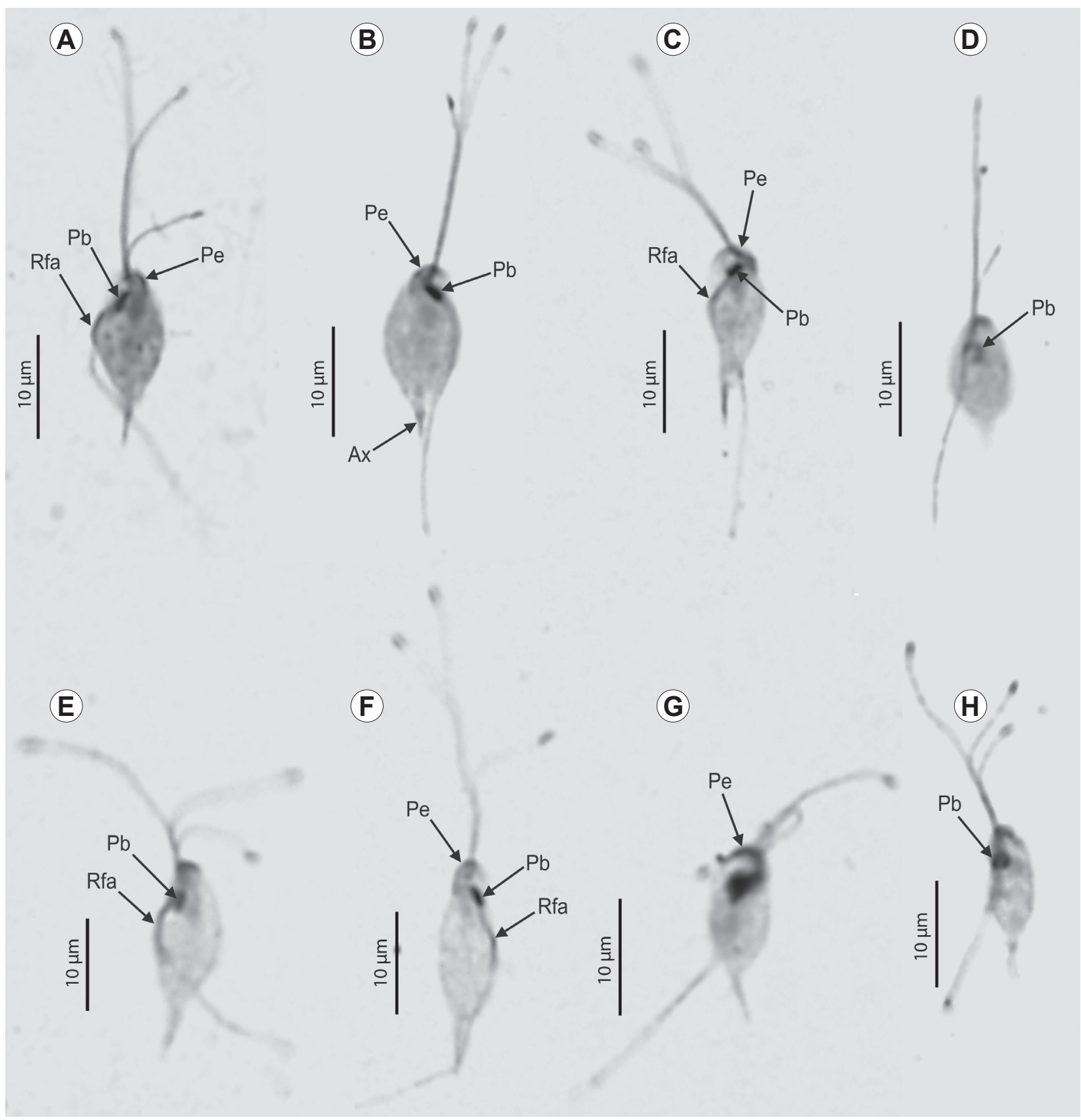

Fig. 2. Protargol-stained specimen of Simplicimonas similis strain CH394. A-D - cell growing in Dobell and Laidlaw's biphasic medium; E-H - cells growing axenically in TYM. Abbreviations: Ax - axostyle; $\mathrm{Pb}$ - parabasal body; $\mathrm{Pe}$ - pelta; $\mathrm{RFa}$ - adhering portion of the recurrent flagellum.

dium (50 cells growing in each medium were measured). The cells growing in the former medium were 5.2-7.9 $(6.3 \pm 0.6) \mu \mathrm{m}$ long and 3.5-5.0 (4.2 \pm 0.4$) \mu \mathrm{m}$ wide (including the pelta and excluding the projecting part of the axostyle), while flagellates growing axenically in TYM were more slender, being 5.5-7.8 (6.5 \pm 0.6$) \mu \mathrm{m}$ long and $2.3-3.9(3.3 \pm 0.3) \mu \mathrm{m}$ wide. These data indicate that the cell width of trichomonads from the genus Simplicimonas is dependent on culture conditions and should not be used for species determination. The other examined characters were stable regardless of culture medium used. The cells possessed typical features of the genus Simplicimonas (Fig. 2), such as three anterior flagella, single recurrent flagellum associated with the cell body, but not forming a well-developed undulating membrane, discoid parabasal body, and Tritrichomonas-type of axostyle (Cepicka et al. 2010). Three unequal anterior flagella were terminated with knobs. The recurrent flagellum adhered by its proximal part to the cell body, the adhesion lacked any undulations and reached one half of the cell. The 'acces- 
sory filament' was not longer than the adherent part of the recurrent flagellum. The parabasal body measured approximately $0.7 \mu \mathrm{m}$ in diameter and was discoid, with a dark margin. The protruding segment of the axostyle was 1.4-3.0 (1.9 \pm 0.3$) \mu \mathrm{m}$ long in Dobell-Laidlaw's medium and 1.5-2.4 (1.9 \pm 0.2$) \mu \mathrm{m}$ long in TYM. Pelta was long and slender.

\section{DISCUSSION}

Diversity of intestinal protists of true bugs is poorly understood and kinetoplastids currently represent the only intensively studied group (Gaunt and Miles 2000, Yurchenko et al. 2008, Votýpka et al. 2010, 2012). Our recent study represents the first attempt to assess the diversity of trichomonads in true bugs. To this end, we employed both culture-dependent and culture-independent methods.

From almost 800 examined specimens of true bugs only three were confirmed to harbour intestinal trichomonads. Based on phylogenetic analysis, the obtained trichomonad isolates CH394, PNG73 and G37 represent three distinct species belonging to the genera Simplicimonas and Monocercomonas Grassi, 1879. On the basis of morphological features, particularly the size and shape of the parabasal body, we assigned the strain CH394 to Simplicimonas similis (see Cepicka et al. 2010). Since the strain PNG73 failed to grow already after the third passage, its morphology could not be examined and its species identity remains uncertain. On the other hand, the uncultured isolate G37 clearly belongs to Monocercomonas colubrorum on the basis of SSU rDNA analysis.

The genera Monocercomonas and Simplicimonas contain multiple species from various hosts including amphibians, reptiles, birds and mammals (Cepicka et al. 2010, Lollis et al. 2011, Dimasuay et al. 2013). Until recently $M$. colubrorum and S. similis have been found only in lizards and/or snakes (Moskowitz 1951, Cepicka et al. 2010, Hampl et al. 2007). Recently, Dimasuay et al. (2013) reported $S$. similis from water buffaloes on the basis of the SSU rDNA sequences. However, the genetic distance between $S$. similis and the trichomonad sequences derived from water buffaloes (GenBank accession numbers KC953858 and KC953859) was 5\% and 6\%, respectively. Such distances are considerably larger than typical intraspecific distances among trichomonads and rather correspond to interspecific distances (Cepicka et al. 2006). Therefore, we assume that the isolates from water buffaloes represent, in fact, a separate lineage, probably a so far undescribed Simplicimonas species distinct from $S$. similis. In contrast, the low genetic distance between our strain CH394 and the type strain of S. similis if both the SSU rDNA and ITS region are considered, justifies the classification of CH394 into S. similis. To the best of our knowledge, this is the first evidence for the occurrence of $M$. colubrorum and $S$. similis in other hosts than reptiles.

Since true bugs and reptiles harbour at least these two common trichomonad species, namely $S$. similis and $M$. colubrorum, their transmission between these hosts is likely to occur. We assume that true bugs may be infected by trichomonads during feeding on reptilian excrements. Coprophagy has been repeatedly observed in various species of true bugs (Constant 2007). Considering the low observed prevalence of trichomonads among true bugs, it seems likely that such transfer occurs only accidentally. Although at this point, we cannot rule out the possibility that reptiles might become infected during predation on true bugs. Indeed, such predation has been already described (Knowlton and Janes 1932, Fuseini 1975, Best and Gennaro 1984).

Since the diversity and mode of transmission of most intestinal trichomonads remain unclear, we cannot reject the possibility that true bugs or other insects serve as vectors of otherwise reptile-specific trichomonads. Since $M$. colubrorum has been reported to be facultatively pathogenic for reptiles (Zwart et al. 1984), its life cycle and potential transmission by predation on insects is worth of further study.

Acknowledgements. This work was supported by the Czech Science Foundation (project P506/11/1317) and Charles University Specific Research Grant No. SVV-2013-267 214, the EU 7th Framework Programme No. 316304 and the Praemium Academiae award to J.L., who is also a Fellow of the Canadian Institute for Advanced Research. We acknowledge the use of research infrastructure that has received funding from the EU 7th Framework Programme, grant agreement No. 316304.

\section{REFERENCES}

Adl S.M., Leander B.S., Simpson A.G.B., Archibald J.M., Anderson O.R., Bass D., Bowser S.S., Brugerolle G., Farmer M.A., Karpov S., Kolisko M., Lane C.E., Lodge D.J., Mann D.G., Meisterfeld R., Mendoza L., Moestrup Ø., Mozley-Standridge S.E., Smirnov A.V., Spiegel F. 2007: Diversity, nomenclature, and taxonomy of protists. Syst. Biol. 56: 684-689.

Best T.L., Gennaro A.L. 1984: Feeding ecology of the lizard, Uta stansburiana, in southeastern New Mexico. J. Herpetol. 18: 291-301.
Brugerolle G. 1973: Sur l'existence de vrais kystes chez les Trichomonadines intestinales. Ultrastructure des kystes de Trichomitus batrachorum Perty 1852, Trichomitus sanguisugae Alexeieff 1911, et Monocercomonas tipulae Mackinnon 1910. C.R. Acad. Sci. Paris 277: 2193-2196.

Cavalier-Smith T. 2002: The phagotrophic origin of eukaryotes and phylogenetic classification of Protozoa. Int. J. Syst. Evol. Microbiol. 52: 297-354.

Cavalier-Smith T. 2003: The excavate protozoan phyla Metamonada Grassé emend. (Anaeromonada, Parabasalia, Carpediemonas, Eopharyngia) and Loukozoa emend. (Jakobea, 
Malawimonas): their evolutionary affinities and new higher taxa. Int. J. Syst. Evol. Microbiol. 53: 1741-1758.

Cepicka I., Hampl V., Kulda J. 2010: Critical taxonomic revision of parabasalids with description of one new genus and three new species. Protist 161: 400-433.

Cepicka I., Hampl V., Kulda J., Flegr J. 2006: New evolutionary lineages, unexpected diversity, and host specificity in the parabasalid genus Tetratrichomonas. Mol. Phylogenet. Evol. 39: $542-551$.

Cepicka I., Kutišová K., Tachezy J., Kulda J., Flegr J. 2005: Cryptic species within the Tetratrichomonas gallinarum species complex revealed by molecular polymorphism. Vet. Parasitol. 128: 11-21.

Constant J. 2007: Note on coprophily and necrophily in the Hemiptera Heteroptera. Bull. Inst. R. Sci. Nat. Belg., Entomol. 77: $107-112$.

DiAmond L.S. 1957: The establishment of various trichomonads of animals and man in axenic cultures. J. Parasitol. 43: 488-490.

Dimasuay K.G.B., Lavilla O.J.Y., Rivera W.L. 2013: New hosts of Simplicimonas similis and Trichomitus batrachorum identified by $18 \mathrm{~S}$ ribosomal RNA gene sequences. J. Parasitol. Res. 2013: 831947.

Dobell C., Laidlaw P.P. 1926: On the cultivation of Entamoeba histolytica and some other entozoic amoebae. Parasitology 18: 283-318.

Dolan M., Wier A.M., Melnitsky H., Whiteside J.H., MarGULIS L. 2004: Cysts and symbionts of Staurojoenina assimilis Kirby from Neotermes. Eur. J. Protistol. 40: 257-264.

FARMER M.A. 1993: Ultrastructure of Ditrichomonas honigbergii n. g., n. sp. (Parabasalia) and its relationship to amitochondrial protists. J. Eukaryot. Microbiol. 40: 619-626.

Fuseini B.A. 1975: Ecology of cotton stainers (Heteroptera: Pyrrhocoridae) in southern Ghana. Biol. J. Linn. Soc. 7: 113-146.

Gaunt M., Miles M. 2000: The ecotopes and evolution of triatomine bugs (Triatominae) and their associated trypanosomes. Mem. Inst. Oswaldo Cruz 95: 557-565.

Hall T.A. 1999: BioEdit: a user-friendly biological sequence alignment editor and analysis program for Windows 95/98/NT. Nucl. Acids Symp. Ser. 41: 95-98.

Hampl V., Cepicka I., Flegr J., Tachezy J., Kulda J. 2007: Morphological and molecular diversity of the monocercomonadid genera Monocercomonas, Hexamastix, and Honigbergiella gen. nov. Protist 158: 365-383.

Katoh K., Misawa K., Kuma K., Miyata T. 2002: MafFT: a novel method for rapid multiple sequence alignment based on fast Fourier transform. Nucl. Acids Res. 30: 3059-3066.

Knowlton G.F., Janes M.J. 1932: Studies of the food habits of Utah lizards. Ohio J. Sci. 32: 467-472.

KowalczyK S.A. 1938: A report on the intestinal protozoa of the larva of the Japanese beetle (Popillia japonica Newm., Coleoptera). Trans. Am. Microsc. Soc. 58: 229-244.

Lollis L., Gerhold R., McDougald L., Beckstead R. 2011: Molecular characterization of Histomonas meleagridis and other parabasalids in the United States using the 5.8S, ITS-1, and ITS-2 regions. J. Parasitol. 97: 610-615.
LuDWIG F.W. 1946: Studies on the protozoan fauna of the larvae of the crane-fly, Tipula abdominalis. I. Flagellates, amoebae, and gregarines. Trans. Am. Micr. Soc. 65: 189-214.

Mackinnon D.L. 1910: New protist parasites from the intestine of Trichoptera. Parasitology 3: 245-254.

MACKInNON D.L. 1912: Protists parasitic in the larva of the cranefly, Tipula sp. Parasitology 5: 175-189.

McElroy S.M., Szalanski A.L., McKay T., Bermudez A.J., Owens C.B., Steelmana C.D. 2005: Molecular assay for the detection of Cochlosoma anatis in house flies and turkey specimens by polymerase chain reaction. Vet. Parasitol. 127: 165-168.

Moskowitz N. 1951: Observations on some intestinal flagellates from reptilian host (Squamata). J. Morphol. 89: 257-321.

NiE D. 1950: Morphology and taxonomy of the intestinal protozoa of the Guinea-pig, Cavia porcella. J. Morphol. 86: 381-493.

Pereira-Neves A., Ribeiro K.C., Benchimol M. 2003: Pseudocysts in trichomonads - new insights. Protist 154: 313-329.

Stamatakis A. 2006: RAxML-VI-HPC: maximum likelihoodbased phylogenetic analyses with thousands of taxa and mixed models. Bioinformatics 22: 2688-2690.

Tachezy J., Tachezy R., Hampl V., Šedinová M., Vañáčová Š., VRlík M., van Ranst M., Flegr J., Kulda J. 2002: Cattle pathogen Tritrichomonas foetus (Riedmüller, 1928) and pig commensal Tritrichomonas suis (Gruby \& Delafond, 1843) belong to the same species. J. Eukaryot. Microbiol. 49: 154-163.

Travis B.V. 1932: A discussion of synonymy in the nomenclature of certain insect flagellates, with the description of a new flagellate from the larvae of Ligyrodes relictus Say (ColeopteraScarabaeidae). Iowa St. Coll. J. Sci. 6: 317-323.

Votý PKa J., Klepetková H., Jirkư M., Kment P., Lukeš J. 2012: Phylogenetic relationships of trypanosomatids parasitising true bugs (Insecta: Heteroptera) in sub-Saharan Africa. Int. J. Parasitol. 42: 489-500.

Votýpka J., Maslov D.A., Yurchenko V., JirkŮ M., Kment P., Lun Z.R., LuKEŠ J. 2010: Probing into the diversity of trypanosomatid flagellates parasitizing insect hosts in SouthWest China reveals both endemism and global dispersal. Mol. Phylogenet. Evol. 54: 243-53.

Yubuki N., Céza V., Cepicka I., Yabuki A., Inagaki Y., Nakayama T., Ionuye I., Leander B.S. 2010: Cryptic diversity of free-living parabasalids, Pseudotrichomonas keilini and Lacusteria cypriaca n. gen., n. sp., as inferred from small subunit rDNA sequences. J. Eukaryot. Microbiol. 57: 554-561.

Yurchenko V.Y., Lukeš J., Tesařová M., Jirkư M., Maslov D.A. 2008: Morphological discordance of the new trypanosomatid species phylogenetically associated with the genus Crithidia. Protist 159: 99-114.

Zhang N., Suh S.O., Blackwell M. 2003: Microorganisms in the gut of beetles: evidence from molecular cloning. J. Invertebr. Pathol. 84: 226-233.

Zwart P., Teunis S.F.M., Cornelissen J.M.M. 1984: Monocercomoniasis in reptiles. J. Zoo. Anim. Med. 15: 129-134.

Accepted 10 January 2014 